\title{
ANTROPOLOGÍA Y EPISTEMOLOGÍAS DEL SUR: EL RETO DE LA DESCOLONIZACIÓN DE LA PRODUCCIÓN DEL CONOCIMIENTO
}

\section{ANTHROPOLOGY AND EPISTEMOLOGIES OF THE SOUTH: THE CHALLENGE OF KNOWLEDGE'S DESCOLONIZATION}

Juan Carlos Gimeno

Universidad Autónoma de Madrid

Ángeles Castaño

Universidad de Sevilla

Los pilares teóricos de la antropología social y cultural desde su institucionalización como disciplina científica en la segunda mitad del siglo XIX han estado ágiles al movimiento. La antropología ha demostrado con el paso del tiempo ser una disciplina articulada con sus contextos sociohistóricos, permeable a los aportes de otras disciplinas y generosa en los diálogos y préstamos, atenta a las transformaciones de los fenómenos sociales que observa y a la vez la producen, y con buena cintura para plegarse a las innovaciones del pensamiento y de la construcción teórica. Esta ductilidad dialéctica le permite pervivir como disciplina con vitalidad, sensible a los procesos globales que afectan al reconocimiento y producción de la diversidad de las sociedades y culturas humanas, y con creatividad aún en la reformulación de sus métodos de obtención y construcción de conocimiento, y en algunos casos, compañera fiel de los olvidados y silenciados en la producción de la historia y diestra para ver donde otras no miran. 
En este número 10 de la Revista Andaluza de Antropología tratamos de abordar dos de los desafíos a los que se enfrenta en este tiempo la producción antropológica: el que se refiere a la dimensión global del mundo (tanto en los procesos que lo producen, en su diversidad, como en la conciencia que se tiene de la misma); y en segundo lugar, el que se relaciona con la crítica epistemológica, donde se da una apertura a formas otras de acceder al conocimiento que discuten y contradicen las epistemologías positivistas. Estos desafíos ponen en cuestión la matriz eurocéntrica de la producción antropológica abriendo la disciplina a la producción de conocimiento desde localizaciones del Sur global (“antropologías del sur”, donde el "sur” es una metáfora de una producción antropológica situada en los márgenes geográficos y sociales).

En los últimos años las críticas poscoloniales y descolonizadoras a la producción del conocimiento social del mundo, en el sentido amplio de ciencias sociales y humanísticas, ha hecho aflorar cuestiones que se ligan a lo que el sociólogo portugués, Boaventura de Sousa Santos (2005), denomina sociología de las ausencias (que permite poner de manifiesto lo silenciado y lo no visto, de manera que la realidad es mucho más amplia que lo considerado existente) y sociología (o ecologías) de las emergencias (cuya agenda parte de formas sociales que ya están aquí, pero no han podido desplegarse por la violencia con la que han sido mantenidas como no existentes (Santos, 2005).

En el fragor de las confrontaciones de la globalización neoliberal y las resistencias que produce, para llenar los vacíos y silencios de un conocimiento social (considerado científico, neutral y objetivo) falto de una actitud dialógica con los conocimientos de los otros agentes y sujetos del "mundo" en que vivimos (considerado tanto un sistemamundo con Wallerstein y Wolf, como una pluralidad de mundos, según nos sugiere la ontología política de Arturo Escobar, 2014), repensar los procesos plurales de producción antropológica se convierte en una tarea apasionante de la que no podemos desentendernos, explorando las encrucijadas de encuentros e invisibilizaciones que caracterizan y producen (selectivamente, según Fabian, 1983) nuestra contemporaneidad.

La relación epistemológica Norte-Sur de preeminencia tradicional unidireccional y asimétrica, se ha quebrado en los últimos años por la deslumbrante irrupción de los encuentros epistemológicos Sur-Sur, cada vez más recurrentes, más factibles y sólidos. Es la otra cara de la globalización. O de la otra globalización. Se trata de poner de relieve las posibilidades alternativas para tomar en serio las vidas de los sujetos (subalternos, colonizados, silenciados) cuya existencia ha sido considerada estando por debajo de lo que Fanon $(2010,1952)$ denomina la línea del ser, y Santos (2010) concibe como la línea abismal.

Esos presentes paralelos al que raras miradas atienden, incluyendo la antropología hegemónica, son los mundos que siguen resistiendo (o r-existiendo en términos de Escobar, 2014) a la colonialidad global de esta fase del neoliberalismo aplastante y erosivo. 
Ante esta potencialidad, las Epistemologías del Sur (Santos y Meneses, 2014) constituyen una propuesta que trata de traducir esos conocimientos ausentes y emergentes, para dialogar con ellos en una propuesta consciente y crítica políticamente transformativa del estatus quo dominante.

En estas tendencias, debates y reflexiones cruzadas, encontradas, entre compañeros y compañeras que habitamos recurrentemente lugares compartidos, se ha gestado la propuesta de este monográfico y su contenido. En septiembre de 2014 en el simposio "Antropología y descolonialidad. Desafíos etnográficos y descolonización de las metodologías", que organizamos en el XIII Congreso Nacional de Antropología, Juan Carlos Gimeno y Ángeles Castaño, en la Universidad Rovira i Virgili en Tarragona, planteamos reflexionar epistemológica y metodológicamente los desafíos que la perspectiva modernidad/ colonialidad/ descolonialidad plantea en los estudios antropológicos y en la producción de conocimiento sobre, con y desde, minorías subalternas y movimientos sociales resistentes a lo largo y ancho del mundo (global). Desde entonces, algunos de nosotros nos hemos encontrado en diversos congresos y seminarios, y hemos recorrido camino juntos. De entre ellos se encuentran algunas de las personas que participan aquí. En muchos de nosotros y nosotras, esta interacción antropológica, ha dejado importantes huellas. Los efluvios y aromas de estos roces y contactos, y estos no son términos inocentes (Meneses, en este número), se expanden desde los textos. ¿Cómo separar el yo cognoscente del antropólogo y antropóloga del ego del sujeto humano que somos con el que se entrelaza en pensamiento, sentimiento y acción en el afán de llegar a un conocimiento mayor y no inocente de los otros? La metodología racional moderna ha afirmado desde presupuestos deshumanizados y desencantados del mundo, con una perspectiva hiper-racional, más propia de dioses que de hombres, que para resolver las tensiones entre un dentro/fuera, un cerca/lejos, y un emic/etic era condición la separación radical y adecuada de estos dos egos, del ciudadano y el científico, para la producción del conocimiento científico.

Los textos que presentamos se han escrito en diálogo con dos de las líneas más significativas en los estudios que se posicionan frente a la colonialidad del conocimiento: la propuesta de las epistemologías del Sur, ylos debates sobre la modernidad/colonialidad/ descolonialidad.

En primer lugar, la propuesta de Paula Meneses con su artículo "Ampliando las epistemologías del Sur a partir de los sabores: diálogos desde los saberes de las mujeres de Mozambique" propone una perspectiva en los estudios etnográficos y de género que abre las posibilidades de situar en el centro a las mujeres africanas, consideradas como no-seres desde la percepción hegemónica del poder/saber, en el modo de atender sus patrimonios, y sobre sus aportaciones y su lugar sociocultural, tanto en el seno de sus sociedades como en el mundo. Apunta al saber de la oratura en torno a la cocina 
y los procesos de preparación de los alimentos en su sentido amplio, como un poder de resistencia cultural, adaptación creativa, agencia y transculturación que pertenece al mundo y al saber de las mujeres mozambiqueñas. En este saber la huella de la historia y la experiencia colonial debe ser tenida en cuenta por la mirada etnográfica, para acceder a los modos, los sentidos y significados con que se expresa y manifiesta esta herencia en las prácticas, los sabores, los olores, los procesos de elaboración y la comensalidad. En esta perspectiva estos saberes situados quedan devueltos a su lugar en igualdad a otros conocimientos, a todos los conocimientos, y nos reclama incluso desde el rol de la investigación la cuestión de la agencia, la diversidad epistemológica y el propio valor de la diversidad cultural de género como un potencial en sí mismo liberador.

En esta perspectiva de ampliar las epistemologías se mueve también el trabajo que presenta Iván Periáñez Bolaño, "Ser y sentir flamenco: descolonizando la estética moderno colonial desde los bordes", centrado en la consideración del flamenco como una epistemología del sentir situada. Haciendo un ejercicio de aesthesis descolonial (Mignolo, 2010), aborda la epistemología del sentir flamenca como un conocimiento vernáculo situado, arraigado en el aprendizaje de saberes orales de transmisión interpersonal y de reproducción grupal. La creación flamenca como proceso de significación supone un corpus de conocimientos en estrecha relación con lo sentido-vivido y resignifica "las heridas coloniales" en el contexto andaluz, español y europeo. En su propuesta, el patrimonio inmaterial del flamenco de grupos subalternizados, es un espacio desde el que se construye sentido en un giro epistemológico, que aleja estas creaciones de las lógicas contemplativas de la obra de arte en su concepción moderna. Así el saber escuchar y el aprender escuchando forman parte de un proceso descentrado de circulación y transmisión de saberes que permite compartir, insertar y expresar las herencias de la subalternidad flamenca articulando los sonidos y el silencio con lo sentido y la memoria. Ubicar el saber del subalterno en el flamenco, y en los procesos actuales que experimenta el flamenco, es una ventana al potencial para la resistencia de este arte en la globalización.

Apuntando a la valorización de los saberes indígenas como fuentes de producción/acceso al conocimiento y la descolonización del pensamiento, Kepa Fernández de Larrinoa, desde el trabajo social comunitario, presenta el artículo: "Paradigma de investigación y pueblos indígenas del primer mundo: Crítica cultural del trabajo social”. En él, trae a debate las propuestas formuladas por docentes e investigadores indígenas adscritos a departamentos de trabajo social en universidades localizadas en países anglosajones con población nativa, en una aproximación denominada "trabajo social indígena". Desde esta perspectiva, que toma en serio la potencialidad de las epistemologías indígenas para la producción de un conocimiento ampliado de la realidad, el autor pone en diálogo las prácticas profesionales del trabajo social en comunidades autóctonas con la creación y transmisión de conocimiento en centros de investigación y educación superior. Fernández 
de Larrinoa discute aspectos teórico-epistemológicos afines al campo de los estudios culturales, poniendo atención a las nociones de la poscolonialidad y subalternidad. Ambos procesos interactúan en la actual reflexión sobre el trabajo social indígena, en base a tres principios: el concepto de competencia cultural; la noción de indigenización del trabajo social; y qué se entiende en el trabajo social indígena por metodologías y técnicas de intervención social descolonizantes.

Recogiendo la creciente producción por parte de intelectuales y académicos indígenas en la producción de investigaciones no colonizadas y descolonizadoras impulsadas en la dos últimas décadas (Smith, 1999) alrededor de lo que se conoce como ciencia nativa (Cagete, 2000) y en relación a los paradigmas de investigación indígena, Fernández de Larrinoa reflexiona sobre la utilidad para el trabajo social de modelos de investigación basados en epistemologías nativas situadas fuera de la aproximación occidental; una investigación que sigue pautas indígenas de producción del conocimiento en relación a las cuales cobra sentido, que se plantea para beneficio de las comunidades indígenas, supeditada a su control y la generación de conocimiento desde los intereses de las mismas. El autor considera estos planteamientos como parte del proceso de descolonización del pensamiento y las prácticas socioculturales y una crítica al trabajo social estandarizado.

El texto de Paula Durán titulado "De la colonización a la revolución social en Túnez. Universalización del saber, conocimiento situado y emancipación científica” aborda el proceso de construcción delas CienciasSociales en Túnez, señalando el papel desempeñado por el conocimiento científico occidental en la consolidación de la colonialidad del sersaber-poder, al objetivar una determinada opción ideológica, que universalizada, se ha convertido en hegemónica, subalternizando otras formas de producir conocimiento no-occidentales. Las formas coloniales de producción del conocimiento occidental son puestas en diálogo con una sociología crítica tunecina que se está enfrentando a nuevos retos tras la revolución social que convulsionó al país en diciembre de 2010. El debate en esta sociología tunecina gira en torno a la pregunta si los paradigmas existentes en las Ciencias Sociales permiten abordar el proceso de transformación que experimenta su sociedad, y en su seno se reivindica una descolonización del conocimiento que atienda la especificidad cultural vernácula, la denuncia de las estructuras jerárquicas, el reconocimiento de un saber endógeno y la reclamación de una simetría de saberes. Durán plantea una ruptura de la dependencia epistemológica occidental, promoviendo un giro decolonial que posibilite la reinvención del conocimiento que, entendido como emancipación a partir del Sur (Santos, 2006), pueda reconocer la justicia cognitiva global entre la diversidad de saberes que coexisten en situación dialógica. En particular, la autora explicita el movimiento intelectual en Túnez por el reconocimiento de la especificidad cultural para la producción del conocimiento sobre la propia realidad social, a través de la búsqueda de una sociología propiamente árabe o de la reivindicación de la islamización 
de las Ciencias Sociales. Un debate que continúa vigente en la actualidad, más polarizado si cabe, como consecuencia de los acontecimientos que ha vivido Túnez en los últimos años.

Bruno Sena Martins, con su artículo "Antropología y poscolonialismo. La memoria postabismal" señala que frente a las formaciones imperiales de las que escribe Stoller (2008) se impone aprender con el Sur y abandonar el dominio de una razón que construye bajo lógicas de dominación, para confrontar las relaciones coloniales que todavía estructuran las relaciones de Occidente con buena parte del mundo. Para ello es necesario asumir que las ciencias sociales, y entre ellas la antropología, necesitan ser descolonizadas, en la medida en que se basan en las prerrogativas y privilegios científicos fundados en la hegemonía global del paradigma económico y cultural de la modernidad occidental. La propuesta poscolonial adquiere pertinencia como una cuestión que se cruza de una manera inevitable con el análisis de contextos marcados por los despojos de la explotación económica y por la demarcación racial perpetrada por los regímenes coloniales. La lucha por el derecho a ser humano es un camino anticolonial totalmente actual. La lucha por gramáticas de dignidad tendrá que cuestionar las formas de ser menos humano que son reproducidas por el universalismo estrecho de los derechos humanos hegemónicos. La alternativa al conocimiento occidental es la atención a las epistemologías del Sur, construidas sobre la crítica de la modernidad occidental desde una teoría de retaguardia que capte la diversidad del mundo, a través de cuatro estrategias: la sociología de las ausencias, la sociología de las emergencias, la ecología de saberes y la traducción intercultural, a través de la que se constituyan nuevas formas de conocimiento e intercomunicación cultural. La traducción intercultural deberá ser incentivada menos como la inauguración de una relación de aprendizaje y más como la propuesta de una relación que se quiere nueva, en el sentido de otro modo de producción de conocimiento.

El artículo, "Desarrollo y colonialidad: una epistemología para el análisis crítico del desarrollismo", presentado por Víctor Bretón y Pablo Palenzuela, proyecta una visión problematizadora de las consecuencias del uso acrítico del posestructuralismo en el campo del desarrollo. Los autores reconocen la importancia de la crítica posestructualista, y la aportación de las perspectivas que se engloban en lo que se ha denominado Modernidad/ colonialidad latinoamericana al poner en evidencia la colonialidad del conocimiento científico en la que se ha sustentado el "complejo desarrollista". Sin embargo, los autores llaman la atención sobre lo que consideran una tendencia de los trabajos posdesarrollistas a desentenderse de las complejidades de las realidades sociales. Utilizando el caso de algunos proyectos en el área andina se presentan dos ámbitos de cuestionamiento, vinculados a un enfoque que bebe en la economía política antropológica. El primero, en relación a las prácticas del buen vivir que se han localizado en la literatura posdesarrollista 
en las comunidades andinas, y son consideradas como alternativas al desarrollo, sin que a juicio de los autores se dé una clara constatación etnográfica de las mismas; en cambio, los autores mantienen la sospecha que tales prácticas puedan representar una apuesta de ciertas elites locales en un nuevo contexto, mas que la puesta en práctica de una agenda política/cultural asumida desde abajo, que es lo que le daría su carácter alternativo. Un segundo cuestionamiento se realiza sobre la dimensión posdesarrollista de las políticas públicas en los países andinos, como Ecuador y Bolivia, donde se ponen de manifiesto las contradicciones entre las declaraciones de principios constitucionales del tipo Buen Vivir y la praxis neo-desarrollista y neo-estatalista de las políticas impulsadas tanto en Ecuador como en Bolivia, que están siendo subrayadas por numerosos analistas, tras un cierto desencanto popular con las esperanzas puestas en las nuevas constituciones.

El artículo de Víctor Bretón y Pablo Palenzuela constituye una defensa del acercamiento etnográfico como aportación específica de la antropología a la comprensión de la complejidad de la realidad social. Pero dicho acercamiento, para los autores, debe producirse por medio de una antropología crítica y comprometida que pretenda, no el conocimiento por el conocimiento mismo, sino un conocimiento para la acción y que contribuya al proceso de descolonización intelectual.

Lourdes Méndez con el artículo, "En Ecuador: decolonialidad, política cultural, y Universidad de las Artes", aborda una crítica de los planes de política cultural diseñados desde 2007 por el Ministerio de Cultura de Ecuador, en los que las nociones de decolonialidad, interculturalidad y Sumark Kawsay (o buen vivir) forman parte del discurso político. La autora destaca como las políticas encubren las praxis y procesos neoliberales del gobierno pos-neoliberal del presidente Correa. El artículo analiza la reflexividad entre agentes académicos y políticos, colocando su foco en la nueva Universidad de las Artes (UARTES). Méndez confronta los discursos políticos y académicos con realidades empíricas, como la reestructuración universitaria en Ecuador y la creación de la UARTES, y llama la atención sobre cómo el uso retórico de dichas nociones encubre las políticas neoliberales desde las que el gobierno de la revolución ciudadana está reestructurando todos los campos de actividad, incluidos los del arte y la cultura. Para la autora, el discurso que disfraza la política real dificulta la formación de una oposición que denuncie estas prácticas. Uno de los síntomas de lo que está ocurriendo sería el cierre de la universidad Amawtay Wasi que revela la fragilidad política en la que se encuentran sumidas las organizaciones indígenas en Ecuador. En este caso se muestra cómo la instrumentalización política de los discursos académicos surgidos de la teoría de la modernidad/colonialidad, lejos de suponer el desarrollo de políticas emancipatorias para los pueblos indígenas minorizados, son utilizados como una vuelta de tuerca en el proceso de desactivación política de sus movimientos y de sus líderes. 
Cambiando de escenario, haciendo converger la agenda académica con las reivindicaciones de autodeterminación de los pueblos y a partir de las inquietudes que le han sido expresadas por hombres y mujeres saharauis durante su acercamiento etnográfico al Sahara Occidental desde 2003, Juan Carlos Gimeno se interroga, en el artículo “Elegir entre paz y justicia? Apuntes para la resolución del conflicto del Sahara Occidental", sobre las posibilidades de resolución del conflicto saharaui a partir de las conversaciones a las que la comunidad internacional llama a las partes, el Frente POLISARIO y el Reino de Marruecos. Se trata de una pregunta producida en un contexto complejo, desde las vidas atropelladas, de mujeres y hombres saharauis, por una violencia que les mantiene en el exilio en los campos de refugiados al sur de Argelia, o bajo la represiva ocupación marroquí y que permanece impune gracias a la complicidad indiferente de la comunidad política internacional y de la opinión pública mundial. Para Gimeno el conflicto del SO muestra la persistencia de la línea abismal (Santos 2010) entre Europa y África, que posibilita al otro lado de la línea (en el Sur global, en África y en el Sahara occidental, en concreto) situaciones de violencia sobre poblaciones y pueblos (aquí el pueblo saharaui) que no se permitirían a este lado de la línea (en el Norte global). En esta situación de colonialidad global el autor reflexiona sobre las condiciones no-imperiales (Santos, 2010) en las que una conversación entre las partes conduciría a una salida del conflicto basada en la justicia y en el respeto a la legalidad internacional.

Finalmente, Ángeles Castaño, en el artículo "Colonialidad interna y europeidad en la política para la inmigración en Andalucía", aborda las políticas integrales para la inmigración comoperformances dela colonialidad del poderen esta comunidad autónoma. Estas políticas regionales se articulan con la dimensión transnacional de las políticas de inmigración europeas que construyen las periferias mediterráneas como Frontera Sur de Europa, y en las que Andalucía acaba incardinándose en los ejes establecidos como territorio que es a la vez periferia del Estado español y territorio fronterizo de la UE. Para la autora, estas políticas construyen un imaginario de la inmigración que atribuye la idea de diversidad cultural en Andalucía al proceso inmigratorio, y se teje en los discursos políticos sobre la interculturalidad que sustituyen a los del multiculturalismo. Castaño destaca la funcionalidad performativa de discursos elaborados en el anti-racismo, que se ha proyectado sobre el ámbito de intervención del tercer sector, y sobre la producción de conocimiento sobre las migraciones. Con este análisis articulado en las propuestas de la teoría descolonial, el análisis crítico del discurso y la crítica literaria posestructuralista la autora pone en evidencia la ideología de la colonialidad subyacente en los discursos de poder, y el modo en que se articulan en intertextualidad con los enunciados de las políticas que se desarrollan en distintos ámbitos de actuación. Se construye así el imaginario de frontera de Andalucía desde un horizonte desarrollista. 


\section{BIBLIOGRAFÍA.}

Cagete, Gregory 2000) Native Science. Natural laws of interdependence. New Mexico: Clear Light Books.

Escobar, Arturo (2014) Sentipensar con la tierra. Nuevas lecturas sobre el desarrollo, territorio y diferencia. Universidad Autónoma Latinoamericana UNAULA: Medellin.

Fanon, Frantz $(2010,1952)$ Piel Negra, Máscaras Blancas. Akal: Madrid.

Johanes, Fabian (1983) Time and the other. How Anthropology Makes Its Object. New York: Colombia University Press.

Mignolo, Walter (2010) "Aesthesis descolonial”, Calle 14, Revista de Investigación en el campo del arte, no 4 , Bogotá.

Santos, Boaventura de Sousa (2005) El milenio huérfano. Madrid: Trotta.

Santos, Boaventura de Sousa (2006) Conocer desde el Sur. Para una cultura política emancipatoria. Lima: Fondo Editorial de la Facultad de Ciencias Sociales - UNMSM.

Santos, Boaventura de Sousa (2010) Para descolonizar Occidente. Más allá del pensamiento abismal. Buenos Aires: Consejo Latinoamericano de Ciencias Sociales-CLACSO; Prometeo Libros.

Santos, Boaventura de Sousa y Meneses, María Paula (eds.) (2014) Epistemologías del Sur (perspectivas). Madrid: Akal.

Smith, Linda T. (1999) Decolonizing methodologies: research and indigenous peoples. Londres: Zed Books.

Stoller, Ann Laura (2008) “Imperial Debris: Reflections on ruins and ruination”. Cultural Anthropology, 23(2), pp. 191-219. 